\title{
Non-referenced Quality Assessment of Image Processing Methods in Infrared Non-destructive Testing
}

\author{
Thomas J. Ramírez-Rozo ${ }^{1}$, Hernan D. Benítez-Restrepo ${ }^{2}$, \\ Julio C. García-Álvarez ${ }^{1}$, and German Castellanos-Domínguez ${ }^{1}$ \\ 1 Universidad Nacional de Colombia, Km 9 Via al Aeropuerto, Manizales, Colombia \\ tjramirezr@unal.edu.co, jcgarciaa@bt.unal.edu.co, \\ cgcastellanosd@unal .edu.co \\ 2 Pontificia Universidad Javeriana, Calle 18 No. 118-250 Av. Cañasgordas, \\ Cali, Colombia \\ hbenitez@javerianacali.edu.co
}

\begin{abstract}
Infrared Non-Destructive Testing (IRNDT) uses several image processing techniques to enhance visual contrast and visibility of defects in inspected materials. The benchmarking of these techniques is often too qualitative due to a lack of quantitative criteria allowing to assess the qualities of the compared methods. In this work, we compare image processing techniques in IRNDT with a non-referenced (NR) image quality assessment (IQA) algorithm. Furthermore, we validate the NR IQA approach through a human-based quality evaluation and analyze statistical properties of IRNDT images. The results show a high correlation between NR IQA measure quality predictions and subjective evaluation. Moreover, the analysis evidenced a relationship of perceived image quality with 1) the spatial power spectral density, and 2) marginal and joint distributions of wavelet coefficients. This analysis provides a quantitative alternative when comparing image processing methods in IRNDT and can be used to develop specific IQA measure for IRNDT.
\end{abstract}

Keywords: Blind quality assessment, image quality, infrared non-destructive testing, natural scene statistics.

\section{Introduction}

Non-Destructive Testing (NDT) refers to those methods that inspect an object, avoiding affection of its basic nature or impairment for its future usefulness. The Infrared NDT (IRNDT) is a non-contact inspection tool which analyzes the heat emitted by objects for fast evaluation of wide surfaces. It is useful to characterize defects such as material cracks, voids and discontinuities. To increase the visibility of these defects, diverse image processing techniques are used in IRNDT to enhance the image contrast. In essence; the performance of such techniques implicitly involves the Image Quality Assessment (IQA), which is 
commonly subjective [10]: the experts determine the state of the defect, depending on the quality of the processed image. The Contrast-based Signal to Noise Ratio (CSNR) is a common measure in IQA which compares IRNDT processing techniques [13, 14, 6]. This measure is defined as the ratio of the absolute contrast at the center of the defect to the spatial noise in a non-defective region in the inspected material sample.

CSNR is considered as a Non-Referenced (NR) measure, since information is distributed in the form of relative contrast and spatial noise in a non-defective region. This feature is required in assessing the visibility of one particular defect. Nevertheless; CSNR is constrained by the existence of a non-defective area. Therefore, definition of a region free of defects in a sample material remains a difficult problem when considering a real structure. In a wide sense, its location is not precisely identified since it may not be known in advance the location of defects, if present at all. Moreover, CSNR is only useful to measure the detectivity of one defect in the image, but it does not provide an assessment of the overall image contrast enhancement provided by the specific technique [1, [8]. The situation previously described indicates that the benchmarking of image processing techniques in IRNDT remains difficult.

Besides, previous work in IQA measures for comparison of image processing techniques in IRNDT employs full-reference measures. In this approach the temperature image (i.e., the acquired image by a IR camera) is the reference image [2]. This study defines infrared image quality metric index (IRIQI) and gradient infrared image quality index (GIRIQI), both based on the universal quality in$\operatorname{dex}(\mathrm{UQI})$ and feature similarity index (FSIM), respectively [17, 19. The main drawback of IRIQI and GIRIQI is their dependence on a reference image and constants to avoid instabilities in the indexes. There is no clear procedure so far to estimate these constants. To the extent of our knowledge, there are no studies about the agreement between IQA measures with human quality judgments. Therefore, the main contributions of this work are: $i)$ Study of the statistics of IR images in IRNDT; $i \boldsymbol{i}$ ) Comparison of image processing techniques in IRNDT by using a NR IQA measure, iii) Analysis of the agreement between the outcomes of a NR IQA measure and subjective quality assessment.

We believe that this work can serve as a solid starting point for further development of IQA measures specially tailored to IRNDT and provides guidance in design of IQA measures for specific fields of image processing. The remainder of this article is organized as follows. Sections 2 and 3 present the image-processing techniques and NR IQA measures, its related results and discussion. Finally, Section 4 concludes the paper.

\section{Image Quality Assessment on Pulsed Thermography- Based Techniques}

Pulsed Thermography (PT) is one of the most common thermal stimulation methods used in IRNDT. Acquired images from PT are records of temperature decay after briefly heating of the test object. The evaluation is carried out on 
the quality measurement of temperature images filtered by using polynomial fitting methods [3], 8]. In this section, we analyze these IRNDT image processing techniques and the NR-IQA measure used for evaluation.

- Thermographic Signal Reconstruction (TSR) enhances the image sequences resulting from pulsed thermography experiments, making use of the one-dimensional heat diffusion equation describing the surface temperature evolution in a semi-infinite sample after thermal stimulation by a Dirac pulse: $T=Q / e \sqrt{\pi t}$, where $t$ is the time, $e$ is the material effusivity and $Q$ is the energy density at the surface. This relationship can be rewritten in a double logarithmic form. So, time dependency of temperature at each pixel can be approximated with a polynomial having the following form: $\ln [T(t)]=a_{0}+a_{1} \ln (t)+a_{2} \ln ^{2}(t)+\ldots+a_{N} \ln ^{N}(t)$. Typically, $N$ is set to 4 or 5 to avoid ringing artifacts and ensure a valid correspondence between fitting accuracy and signal de-noising for different IRNDT applications. The qualitative results of TSR adequately allow the detection of defects, the reduction of data for processing and filtering of high-frequency noise [16. Time derivatives of fitted polynomial are useful for thermal analysis as well. The first and second-time derivative for the Nth degree polynomial can be expressed as: $d l n[T(t)]=\sum_{n=1}^{N} n a_{n} \ln ^{n-1}(t)$ and $\frac{d^{2}}{d t^{2}} \ln [T(t)]=\sum_{n=2}^{N}(n-1) a_{n} \ln ^{n-2}(t)$, respectively.

- Normalized Thermal Contrast (NTC) is of interest in IRNDT for defect visibility evaluation, image quality enhancement, and calculation of parameters such as the depth and the thickness [9]. Normalized thermal contrast $N C(t)$ is computed with respect to the values of temperature at the instant $t_{\mathrm{m}}$ when the excess temperature is maximal (depending on the heating stimulation). Normalization may also be done with values at the end of the thermal process (i.e time $t_{\mathrm{e}}$ ). So, $N C(t)=\left[T_{\mathrm{def}}(t) / T_{\mathrm{def}}\left(t_{\mathrm{m}}\right)\right]-$ $\left[T_{\text {no-def }}(t) / T_{\text {no-def }}\left(t_{\mathrm{m}}\right)\right]$ where $T_{\text {def }}(t)$ and $T_{\text {no-def }}(t)$, are time temperature curves in defective and non-defective regions of the material.

\section{Experimental Setup and Results}

\subsection{Image Database Setup}

The sequence of acquired images comes from the PT inspection of a Carbon Fiber Reinforced Plastic (CFRP) sample with 25 defects at different depths and sizes, depicted in Fig. 1a Defects are simulated with Teflon inserts. For this test, an infrared camera Santa Barbara Focal Plane SBF125 with sensor size $320 \times 256$ acquires the images at the spectral band $[3,5] \mu \mathrm{m}$ with a sampling frequency of $157.3 \mathrm{~Hz}$. The size of temperature images' sequence is $292 \times 246 \times 991$. Resulting image sequence is labeled as acquired. It is thereafter processed by each following technique: TSR (filtered temperature images), first derivative, second derivative, NTC and filtered NTC method. Finally, each technique provides a different sequence, labeled by the same technique's name. 


\subsection{NR IQA and Human-Based Evaluation}

The main purpose of the evaluation in the field of IRNDT is to assess a variety of image-processing techniques. The main drawback is that there is no ground truth image in the same domain of the image to be analyzed. For this objective, we use two NR measures for the perceptual evaluation.

- First, the Distortion Identification-based Image Verity and INtegrity Evaluation (DIIVINE) is a non-reference image quality measure, extracting natural scene statistics of a diverse kind of images. This index is not straitened for a particular distortion type, but can predict the type of distortion of an image, providing a related score. This approach is based on the hypothesis that the natural scenes possess certain statistical properties, which are altered in the presence of a distortion, making them un-natural 11]. Accordingly; DIIVINE index is computed for each sequence produced by the analyzed image-processing techniques. Figure $1 \mathrm{~b}$ shows smoothed DIIVINE curves, indicating the effect of the polynomial fitting enhancement of the images. For all the curves, minimum DIIVINE values appear at their onsets, in agreement with the thermal transient response at the PT process. Hence, the visual contrast of defects is high at early frames since the effects of 3-D conduction in the sample are not so remarkable at these times.

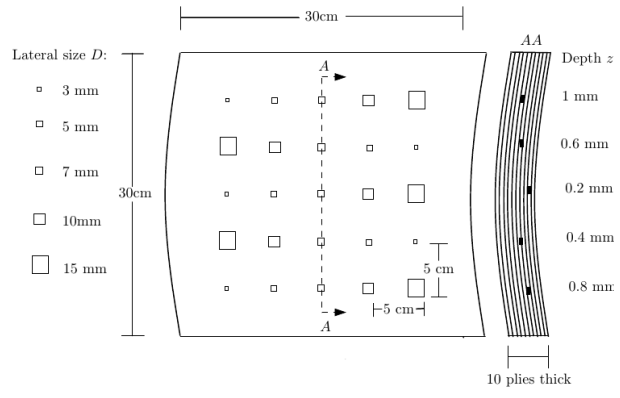

(a) CFRP sample

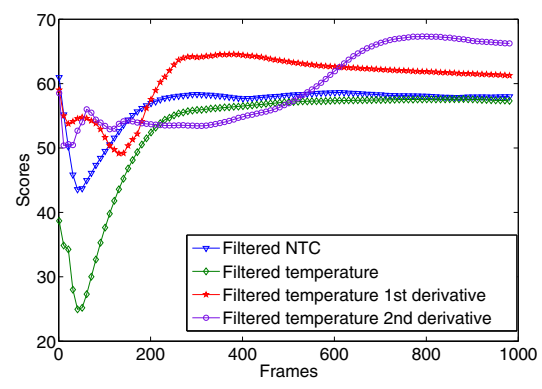

(b) DIIVINE scores

Fig. 1. 1a CFRP Sample, 1b DIIVINE scores for images belonging to each processed sequence

- The second measure is based on human perception, performed as a subjective quality judgment. This comparison is based on previous studies presented at [15] and [18. For this purpose, we use a subset of the image sequences, namely selected database. This collection has 18 images, selected as follows: We extract three images from each sequence (filtered temperature, first derivative, second derivative, NTC, filtered NTC and acquired temperature). Since each image of every different sequence has a DIIVINE scores, the selection criteria is chosen by their DIIVINE score. As a result, we obtain the Best, Worst and Mean DIIVINE scores. 
The size of each image is $292 \times 246$. We arbitrary re-size (using bicubic interpolation) these images to $584 \times 492$ for a proper visualization during the subjective evaluation. For each one of the independent sessions, the number of subjects participating in the perceptual test are 21 and 38, respectively. The test was accomplished in a computer roon 1 . The subject pools consisted in students without experience about image quality assessment in IRNDT and image distortions. The subjects were not tested for vision problems. Each subject was individually briefed about the criteria to evaluate images. The subjects reported their judgment by dragging a sliding bar on a quality scale. The scale of the sliding bar was unmarked numerical, instead the use of labels equidistant with adjectives was used: "Bad", "Poor", "Fair", "Good" and "Excellent". The values obtained belong to 0-100 interval. Both sessions had a duration less than 13 minutes in order to avoid the fatigue in the subjects. The experiments were conducted with identical Microsoft Windows work stations using a GUI Matlab interface. The workstation was placed in an office environment with normal indoor illumination. Same display settings were used for each subject in each session. Subjects viewed the monitors from an approximated distance of 2-2.5 displays width.

Subsequently, we compute the Mean Opinon Score (MOS), being this, the result of the perceived image quality test. In order to validate the results obtained from the MOS, the experiment is deployed in two sessions: the former is composed by images of filtered temperature (TSR), first derivative and second derivative. The second part is composed by NTC, Filtered NTC and acquired temperature images. The scores of both sessions pass through the outlier elimination process. Thereafter, variation of the standard deviation of scores of each session is observed. If this variation is not significant, scores of respective sessions can be validated. We report the comparison between MOS and DIIVINE in Table 1 .

For the selected database, we obtain a low correlation coefficient (Pearson 0.56 and Spearman 0.68). We observe that images corresponding to bestTSR, worst-TSR, mean-TSR, best-NTC and worst-Acquired have DIIVINE scores farther than one standard deviation from corresponding MOS. So, we remove those images, improving the correlation coefficients (Pearson 0.81, Spearman 0.79). Finally, we remove the outlier MOS those scores outside of 2 standard deviations about the MOS mean score, obtaining higher correlation results (Pearson 0.84, Spearman 0.83). Each sequence of images has 991 frames. To have MOS for each image in the sequences takes a lot time and attention from evaluated subjects. Hence, we decided to extract and evaluate with MOS those images with the lowest (high image quality), highest (low image quality) and mean DIIVINE value for each sequence. This simplifies the analysis of correlations between DIIVINE and MOS.

\footnotetext{
${ }^{1}$ Computing laboratory at Universidad Nacional de Colombia, Manizales.
} 
Table 1. DIIVINE and MOS values on the images of Selected database

\begin{tabular}{|c|c|c|c|c|}
\hline Technique & Quality & IIVINE & $\operatorname{MOS} \pm$ std & erence $(\%)$ \\
\hline Filtered temperature & $\begin{array}{c}\text { Best } \\
\text { Worst } \\
\text { Mean }\end{array}$ & $\begin{array}{l}25.01 \\
57.64 \\
52.81\end{array}$ & $\begin{array}{l}49.48 \pm 24.94 \\
98.31 \pm 16.79 \\
67.69 \pm 22.90\end{array}$ & $\begin{array}{l}24.47 \\
40.67 \\
14.88\end{array}$ \\
\hline Filtered temperature 1st derivative & $\begin{array}{c}\text { Best } \\
\text { Worst } \\
\text { Mean }\end{array}$ & $\begin{array}{l}35.29 \\
61.72 \\
48.89\end{array}$ & $\begin{array}{l}41.76 \pm 20.70 \\
71.70 \pm 21.36 \\
64.41 \pm 21,92\end{array}$ & $\begin{array}{c}6.47 \\
9.98 \\
15.52\end{array}$ \\
\hline Filtered temperature 2nd derivative & \begin{tabular}{|c|} 
Best \\
Worst \\
Mean
\end{tabular} & $\begin{array}{l}41.71 \\
52.35 \\
48.00 \\
\end{array}$ & $\begin{array}{l}50.99 \pm 22.87 \\
47.38 \pm 27.20 \\
42.09 \pm 21.10\end{array}$ & $\begin{array}{l}9.28 \\
4.98 \\
5.91 \\
\end{array}$ \\
\hline NTC & $\begin{array}{c}\text { Best } \\
\text { Worst } \\
\text { Mean }\end{array}$ & $\begin{array}{l}51.17 \\
78.23 \\
62.46\end{array}$ & $\begin{array}{l}28.78 \pm 31.00 \\
70.46 \pm 26.03 \\
58.12 \pm 23.16\end{array}$ & $\begin{array}{c}22.39 \\
7.77 \\
4.34\end{array}$ \\
\hline Filtered NTC & $\begin{array}{c}\text { Best } \\
\text { Worst } \\
\text { Mean }\end{array}$ & $\begin{array}{l}45.95 \\
63.96 \\
59.37\end{array}$ & $\begin{array}{l}44.86 \pm 27.20 \\
74.62 \pm 27.61 \\
65.37 \pm 29.41\end{array}$ & $\begin{array}{c}1.09 \\
10.66 \\
5.99\end{array}$ \\
\hline Acquired temperature & $\begin{array}{c}\text { Best } \\
\text { Worst } \\
\text { Mean }\end{array}$ & $\begin{array}{l}44.91 \\
57.76 \\
31.32\end{array}$ & $\begin{array}{c}48.29 \pm 15.04 \\
90.48 \pm 7.47 \\
42.83 \pm 11.74\end{array}$ & $\begin{array}{c}3.39 \\
32.72 \\
11.51\end{array}$ \\
\hline
\end{tabular}

\subsection{Statistics of IRNDT Images}

Since DIIVINE is based on scene statistics which govern the behavior of natural images, a statistical analysis is important to describe the nature of IRNDT images. This nature can be described by spatial power spectral density (PSD). In natural images PSD follows the power law, since it decays at a rate $1 / f^{\alpha}$ where $f$ is the spatial frequency and $\alpha$ is an adjustment parameter which depends on the image model [5]. Figure 2 presents acquired temperature images that we arbitrary chose, according to the measured DIIVINE value. By this regard, these images are labeled as Best, Worst and Mean DIIVINE images.

Figure 3 shows the spatial PSD on log-log axes of acquired temperature images in Fig. 2. Spatial PSD of these images is large at low frequencies and decreases at high frequencies. To establish if these images follow a power law distribution we compute $\alpha$ and a $p$-value for the fitted power-law model at each image. The estimation of these parameters was made according to methodology proposed by Clauset et al in [4. We find that for acquired temperature sequence, the image with best DIIVINE index obtained a $p$-value $=0.94$ and worst DIIVINE index obtained a $p$-value $=0$. According to [4], if the resulting $p$-value is greater than 0.1 , the power law is then a plausible hypothesis for the spatial PSD. Thus, we conclude that temperature images with good quality, according to DIIVINE outcomes, follows a power law distribution while images with bad quality present no power law distribution.

Marginal Distributions: We first present the results and analysis of the marginal wavelet distributions. The Haar wavelet filter is selected to obtain the detail coefficients for two decomposition scales. Figure 4 shows a comparison of the marginal statistics of wavelet coefficients across horizontal and vertical orientations at two scales for acquired temperature images at Fig. 2 . 


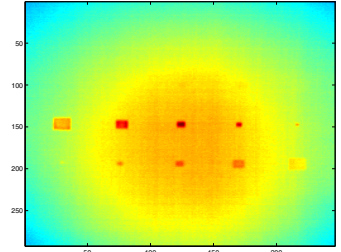

(a) Best DIIVINE score value

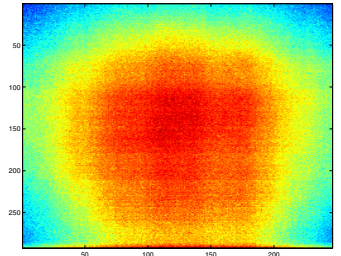

(b) Worst DIIVINE score value

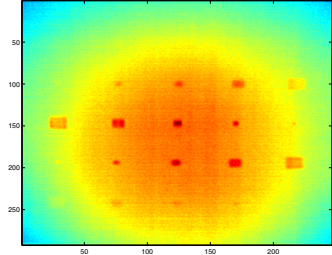

(c) Mean DIIVINE score value

Fig. 2. Acquired temperature images

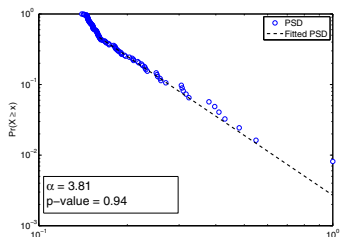

(a) Best DIIVINE score.

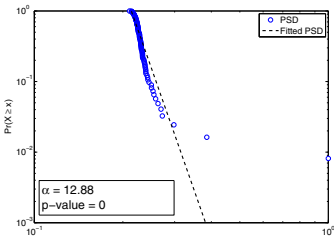

(b) Worst DIIVINE score.

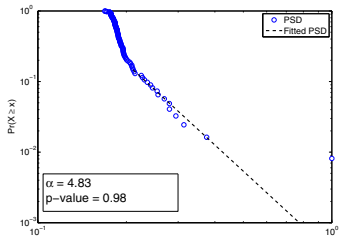

(c) Mean DIIVINE score.

Fig. 3. Power Spectra Density estimated for acquired temperature images and its corresponding fitting

Table 2. Statistical moments of detail wavelet coefficients at different scales, for Acquired Temperature images

\begin{tabular}{c|c|c|c|c|c}
\hline \multirow{2}{*}{ Selected image } & Wavelet Coefficients & \multicolumn{2}{|c|}{$\begin{array}{c}\text { First Scale } \\
\text { Skewness }\end{array}$} & \multicolumn{2}{c}{$\begin{array}{c}\text { Second Scale } \\
\text { Kurtosis }\end{array}$} \\
\hline \multirow{2}{*}{ Best } & Horizontal Detail & 2.93 & 10.47 & 3.31 & 13.23 \\
& Vertical Detail & 2.49 & 7.93 & 2.78 & 9.62 \\
\hline \multirow{2}{*}{ Worst } & Horizontal Detail & 0.80 & 2.13 & 0.89 & 2.28 \\
& Vertical Detail & 0.77 & 2.05 & 0.67 & 2.02 \\
\hline \multirow{2}{*}{ Mean } & Horizontal Detail & 1.36 & 3.34 & 1.93 & 5.34 \\
& Vertical Detail & 0.77 & 2.04 & 1.53 & 4.17 \\
\hline
\end{tabular}

From this Figure, the distribution curves for acquired temperature images with Best and Mean DIIVINE scores exhibit significantly stronger kurtosis (i.e peakedness) than the acquired temperature image with Worst DIIVINE, numerically confirmed in Table 2, Besides, there are high kurtosis values, for the images corresponding to best and mean DIIVINE scores, across scales in vertical and horizontal directions.

High kurtotic distributions reflect specific intuitive properties of images of the real world. Such highly kurtotic distributions have important implications with respect to the sensory neural coding of natural scenes [7].

Joint Distributions: The joint distribution of wavelet coefficients statistically analyze these images, by measuring wavelet values with respect to orientation, 


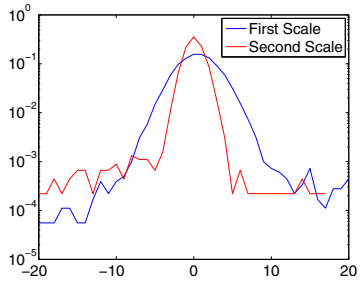

(a) Best DIIVINE score value

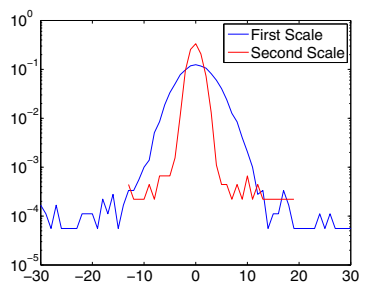

(d) Best DIIVINE score value

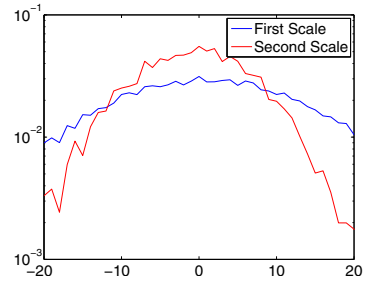

(b) Worst DIIVINE score value

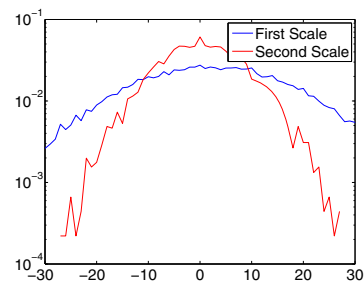

(e) Worst DIIVINE score value

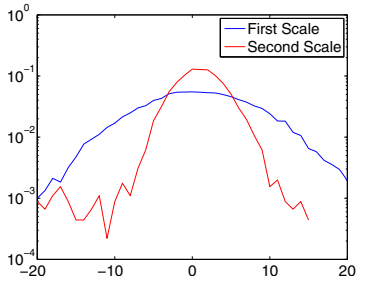

(c) Mean DIIVINE score value

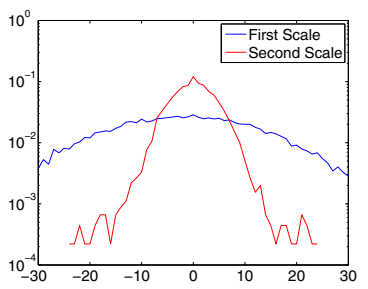

(f) Mean DIIVINE score value

Fig. 4. Acquired temperature images Marginal horizontal wavelet coefficients distribution (Top), Marginal vertical wavelet coefficients distribution (Bottom), first and second scale

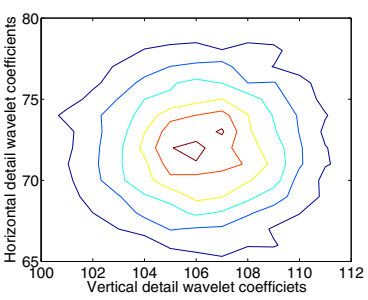

(a) Best DIIVINE score value

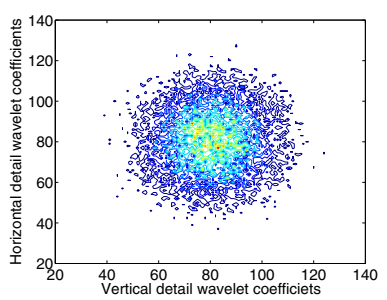

(b) Worst DIIVINE score value

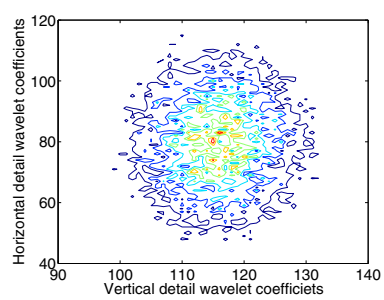

(c) Mean DIIVINE score value

Fig. 5. Joint horizontal vs. vertical wavelet coefficients histograms

sub-band and spatial indexes. This measure comprises the histogram for vertical orientation coefficients versus horizontal orientation coefficients is computed according to definition provided in [12]. Figure 5 shows the contour plots of the log probability for joint distributions of vertical and horizontal wavelet coefficients extracted from images in Fig. 2, There is a noticeable difference between joint histograms: the joint histogram of the image with the best DIIVINE score presents an ellipsoidal shape; on the contrary, images with worst and mean DIIVINE scores display contour surfaces with irregular shapes that do not contain information about dependencies between orientations. 


\section{Conclusion}

This work presents an IQA-based evaluation of image processing techniques in IRNDT. We compared the following techniques: filtered temperature, first and second derivatives profiles, normalized thermal contrast and acquired temperature. The comparison is carried out by the NR IQA measure termed DIIVINE index, and mean opinion scores coming from human evaluations. We detailed the PSD, marginal and joint distributions of wavelet coefficients as approaches to understand the statistics of temperature images in IRNDT. From this study, we conclude that the spatial PSD of those temperature images with "good" quality, according to DIIVINE scores, fits to a power law distribution. Moreover, their marginal distributions have high kurtosis across subbands and orientations. Furthermore, their joint distribution exhibits an ellipsoidal shape indicating a Gaussian distribution of wavelet coefficients. In addition, we confronted the DIIVINE predictions against human quality judgments represented by MOS. We found that DIIVINE correlates with subjective image quality assessment. However, this evaluation of IRNDT images is difficult since the subjects who participated in the study are not familiarized with this type of images. In the same way, DIIVINE is designed for particular distortion types tailored for visible spectra, as a consequence, it fails to assess properly some images, where there are no visible defects at all. According to Table 1. DIIVINE predicted values and its correlation with human quality judgment, the best image processing technique is first derivative, the criteria used to conclude this statement is based on the fact that DIIVINE against MOS has high correlation values, as well as low scores, which means "good" image quality. Further work shall involve the analysis of the statistics of transformed temperature images and the study of the specific distortions that affect processed images in IRNDT to propose a NR IQA measure specifically designed for IRNDT.

Acknowledgements. The authors acknowledge to Colciencias and the Universidad Nacional de Colombia for the financial support of the research projects "Sistema autónomo de monitoreo de vibraciones para diagnóstico de fallas no estacionarias" (with code 1101-521-28792). The authors also thank Canada Research Program (CRC): Multipolar Infrared Vision Canada Research Chair (MiViM) for providing the CFRP sample.

\section{References}

1. Balageas, D.L.: Defense and illustration of time-resolved pulsed thermography for nde. Quantitative Infra Red Thermography Journal 9(1), 3-32 (2012)

2. Benítez, H.D.: Objective Image Quality Assessment in infrared non-destructive testing. In: XVII Symposium of Image, Signal Processing, and Artificial Vision, STSIVA (2012)

3. Castanedo, C.: Quantitative subsurface defect evaluation by pulsed phase thermography: depth retrieval with the phase. Ph.D. thesis, Université Laval (2005) 
4. Clauset, A., Shalizi, C.R., Newman, M.E.: Power-law distributions in empirical data. SIAM Review 51(4), 661-703 (2009)

5. Deriugin, N.G.: The power spectrum and the correlation function of the television signal. Telecommunications 1(7), 1-12 (1956)

6. Duan, Y., Servais, P., Genest, M., Ibarra-Castanedo, C., Maldague, X.P.V.: ThermoPoD: A reliability study on active infrared thermography for the inspection of composite materials. Journal of Mechanical Science and Technology 26(7), 1985-1991 (2012)

7. Field, D.J.: What is the goal of sensory coding? Neural Computation 6(4), 559-601 (1994)

8. Ibarra-Castanedo, C., Piau, J.M., Guilbert, S., Avdelidis, N.P., Genest, M., Bendada, A., Maldague, X.P.V.: Comparative study of active thermography techniques for the nondestructive evaluation of honeycomb structures. Research in Nondestructive Evaluation 20(1), 1-31 (2009)

9. Maldague, X.: Introduction to NDT by active infrared thermography. Materials Evaluation 6(9), 1060-1073 (2002)

10. Maldague, X.: Theory and practice of infrared technology for nondestructive testing. New York (2001)

11. Moorthy, A.K., Bovik, A.C.: Blind image quality assessment: From natural scene statistics to perceptual quality. IEEE Transactions on Image Processing 20(12), 3350-3364 (2011)

12. Morris, N.J.W., Avidan, S., Matusik, W., Pfister, H.: Statistics of infrared images. In: IEEE Conference on Computer Vision and Pattern Recognition, CVPR 2007, pp. 1-7. IEEE (2007)

13. Omar, M., Hassan, M., Saito, K.: Optimizing thermography depth probing with a dynamic thermal point spread function. Infrared Physics \& Technology 46(6), 506-514 (2005)

14. Pickering, S., Almond, D.: Matched excitation energy comparison of the pulse and lock-in thermography NDE techniques. NDT and E International 41(7), 501-509 (2008)

15. Sheikh, H.R., Sabir, M.F., Bovik, A.C.: A statistical evaluation of recent full reference image quality assessment algorithms. IEEE Transactions on Image Processing 15(11), 3440-3451 (2006)

16. Shepard, S.M.: Advances in thermographic NDT. In: Infrared Technology and Applications XXIX, vol. 5074, pp. 882-887 (2003)

17. Wang, Z., Bovik, A.: A universal image quality index. IEEE Signal Processing Letters 9, 81-84 (2002)

18. Wang, Z., Bovik, A.C., Sheikh, H.R., Simoncelli, E.P.: Image quality assessment: From error visibility to structural similarity. IEEE Transactions on Image Processing 13(4), 600-612 (2004)

19. Zhang, L., Zhang, L., Mou, X., Zhang, D.: FSIM: A feature similarity index for image quality assessment. IEEE Transactions on Image Processing 20(8), 2378-2386 (2011) 